\title{
Diversidad florística en cimas de la Sierra Madre Occidental, México, y Su Relación con Variables ambientales
}

\author{
Lizeth Ruacho-González' , M. Socorro González-Elizondo, Martha González-Elizondo \\ Y CELIA LÓPEZ-GONZÁLEZ
}

Centro Interdisciplinario de Investigación para el Desarrollo Integral Regional, Instituto Politécnico Nacional, Unidad Durango, Durango, Durango, México

1Autor para correspondencia: liz_3626@hotmail.com

\begin{abstract}
Resumen: Las partes más altas de la Sierra Madre Occidental presentan comunidades vegetales con algunas características de vegetación alpina. Estos sitios son ecológicamente importantes, pues además de sus altos índices de diversidad, son de las primeras zonas terrestres donde pueden detectarse efectos del cambio climático. El objetivo del trabajo fue aportar información acerca de la composición florística y su cobertura en las cimas de tres de los picos más altos de la Sierra Madre Occidental: Cerro Gordo, Huehuento y Las Antenas, comparar su riqueza específica y medir la similitud florística entre las cimas e identificar las variables ambientales que más influyen sobre la vegetación. Los muestreos se basaron en la metodología propuesta por el Proyecto GLORIA. Se realizó un Análisis de Correspondencias Canónicas, el cual mostró una estrecha relación entre la presencia de las especies y las variables de precipitación, temperatura, continentalidad, superficie de afloramiento rocoso, perturbación y altitud. Se registraron 175 taxa y una similitud florística baja, con únicamente dos compartidos entre los tres cerros. Aunque en la cima de Las Antenas fue donde se registró el mayor número de taxa, tanto en el inventario florístico como en las unidades de muestreo, fue en el cerro Huehuento donde se registró el mayor número de especies $/ \mathrm{m}^{2}$. El elemento alpino está escasamente representado en la Sierra Madre Occidental, compuesto por Juniperus blancoi var. huehuentensis, Draba implexa, Micranthes mexicana, Bromus richardsonii y varias especies de Sedum, Galinsoga y Muhlenbergia.
\end{abstract}

Palabras clave: alpino, análisis de correspondencia canónica, flora, riqueza específica, vegetación.

\begin{abstract}
The highest peaks of the Sierra Madre Occidental harbor plant communities with some alpine vegetation features. These sites are ecologically important because, in addition to their high levels of diversity, they are among the first land areas where effects of climatic change can be detected. The objectives of this work were to provide information about plant cover and floristic composition on the summit of three of the highest peaks of the Sierra Madre Occidental: Cerro Gordo, Huehuento and Las Antenas, as well as to compare their species richness, to measure their floristic similarity, and to identify the main environmental variables that may have an influence on vegetation. Sampling was based on the methodology proposed by the GLORIA Project and data were analyzed using Canonical Correspondence Analysis. Results show a close relationship between species presence in these sites and the rainfall, temperature, continentality, density of rock outcrops, disturbance and elevation variables. We recorded 175 taxa, floristic similarity among summits was low, and only two species were shared among the three peaks. Although the highest number of species was recorded at Las Antenas, both, in the floristic inventory and on the sampling plots, it was at Huehuento where the highest number of species $/ \mathrm{m}^{2}$ was recorded. The alpine element is poorly represented in the Sierra Madre Occidental, composed mainly by Juniperus blancoi var. huehuentensis, Draba implexa, Micranthes mexicana, Bromus richardsonii, and some dwarf species of Sedum, Galinsoga, and Muhlenbergia.
\end{abstract}

Key words: alpine, canonical correspondence analysis, flora, richness, vegetation.

$\mathbf{E}$ 1 territorio de México, por su gran biodiversidad ha sido objeto de numerosos trabajos descriptivos y de análisis de la vegetación desde el siglo XIX (Rzedowski, 1978; Morrone, 2005); sin embargo, existen regiones en las cuales falta mucho por estudiar. Ejemplo de ello es la Sierra Madre Occidental (SMO), área de gran importancia ecológica y económica para México (González-Elizondo et al., 2012), particularmente las comunidades vegetales que se encuentran en las partes más altas.

Aunque existen trabajos en los cuales se han reportado elementos de afinidad alpina para tres de los picos más altos de la SMO: Cerro Gordo y Huehuento en Durango (Gonzá- 
lez-Elizondo et al., 2007; Ruacho-González, 2011) y Mohinora en el estado de Chihuahua (Correll, 1960; McDonald, 1993; McDonald et al., 2011), a excepción de la reciente publicación de McDonald et al. (2011) para el cerro Mohinora, no existen inventarios florísticos de estos sitios, por lo que se ignora en qué medida los elementos alpinos están presentes en las cimas de la SMO y qué relación tienen con las características de las mismas.

Se considera de primordial importancia conocer y conservar este tipo de ecosistemas debido a que las partes altas de las montañas son áreas con altos índices de biodiversidad y contienen un considerable número de especies de plantas endémicas (McDonald, 1993; Giménez et al., 2003; Pauli et al., 2003; Villar y Benito, 2003). Asimismo, los biomas de alta montaña son de las primeras zonas terrestres donde pueden detectarse los cambios climáticos (Pauli et al., 2003; Villar y Benito, 2003) gracias a la presencia de ecotonos muy definidos, que facilitan el reconocimiento de las posibles áreas de desplazamiento provocadas por el calentamiento y otras variables resultantes de éste (Pauli et al., 2003; Wieser et al., 2009). En términos generales, la riqueza y diversidad florística de estos sitios está determinada por factores climáticos y edáficos, y además, varía con su ubicación geográfica y la conformación de su cima, factores que determinan el microclima de cada una (Nagy y Grabherr, 2009; Grabherr et al., 2010).

La finalidad de este trabajo fue estudiar la flora y la vegetación de tres de los cerros de mayor elevación en la SMO, con tres objetivos en particular: (1) documentar la composición y cobertura de la flora de las cimas e identificar sus elementos alpinos, (2) comparar la riqueza específica y la similitud florística entre las cimas y (3) identificar cuáles son las principales variables ambientales asociadas con estas comunidades vegetales.

\section{Materiales y métodos}

Área de estudio. Se seleccionaron tres áreas con altas elevaciones en la SMO: Cerro Gordo (23 $12^{\prime} 22^{\prime \prime}$ N, $104^{\circ} 56^{\prime}$ 39" O a 3,347 m s.n.m. en el municipio de Pueblo Nuevo, Dgo.), que representa la cima más alta de la SMO, cerro Huehuento (24 04' 31" N, $105^{\circ} 44^{\prime} 24^{\prime \prime}$ O a 3,262 m s.n.m., en San Dimas, Dgo.) y el cerro Las Antenas en la Sierra El Epazote (24 36' 12" N, 105 06' 37' O a 3,224 m s.n.m., en Canatlán, Dgo. ; Figura 1).

Los tres cerros presentan regosoles y cambisoles sobre un fuerte afloramiento de roca ígnea. La vegetación predominante es bosque mixto con presencia de Abies durangensis Martínez, Pseudotsuga menziesii (Mirb.) Franco y varias especies de los géneros Arbutus, Quercus y Pinus, siendo $P$. rudis Endl. la especie de pino dominante y la que marca el ecotono entre la línea de árboles y las comunidades cimeras.

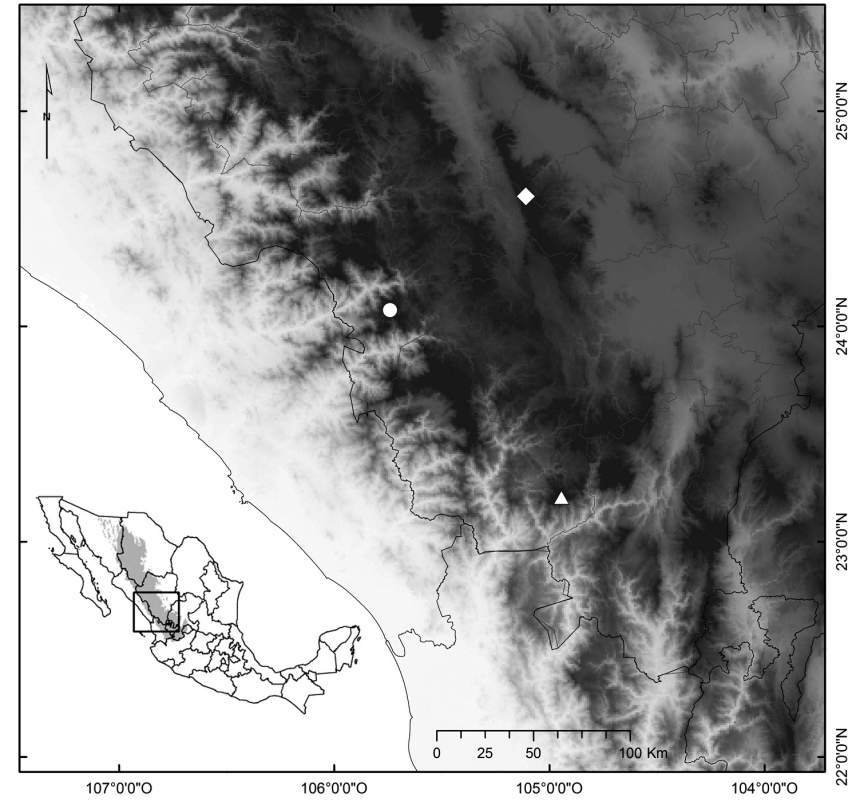

Figura 1. [r1]Área de estudio. $(\triangle)$ Cerro Gordo, $(\bigcirc)$ Huehuento, $(\diamond)$ Las Antenas.

Estimación de la cobertura. El diseño de muestreo se basó en el método propuesto por el Proyecto de Iniciativa para la Investigación y el Seguimiento Global de los Ambientes Alpinos (GLORIA, por sus siglas en inglés, Pauli et al., 2003). Aunque la SMO no cumple con los criterios requeridos para establecer una zona piloto de GLORIA, se optó por usar sus métodos de muestreo, ya que están enfocados en documentar la flora y la vegetación de las cimas de montañas, así como en detectar y dar seguimiento a los cambios en la estructura vegetal.

A diferencia de GLORIA, en la que el diseño de muestreo consta de cuatro parcelas de $3 \times 3 \mathrm{~m}$ en cada cima, el diseño en este trabajo fue de ocho parcelas, dos hacia cada punto cardinal, una por cada una de dos áreas cimeras definidas (5 m y $10 \mathrm{~m}$ de elevación hacia abajo desde la cima). Debido a la pendiente de algunas exposiciones, no fue factible establecer las ocho parcelas en todos los cerros y en total sólo se muestrearon 19, en lugar de las 24 posibles (ocho en Cerro Gordo, cuatro en Huehuento y siete en Las Antenas; Figura 2).

Dentro de cada parcela, en el $\mathrm{m}^{2}$ de cada esquina (Figura 2) se estimó la proporción del área $\left(\mathrm{en}^{\mathrm{dm}} \mathrm{m}^{2}\right.$ ) cubierta por plantas así como de elementos abióticos que cubrían el suelo. Cada uno de los cuadros de $1 \mathrm{~m}^{2}$ de las esquinas representa una unidad de muestreo, para un total de $19 \times 4=$ $76 \mathrm{~m}^{2}$ muestreados (denominados cuadros). El trabajo de campo se realizó durante los meses de septiembre y octubre de 2010.

Inventario de especies (Riqueza): De manera simultánea a los muestreos en cuadros, se llevó a cabo un inventario de 


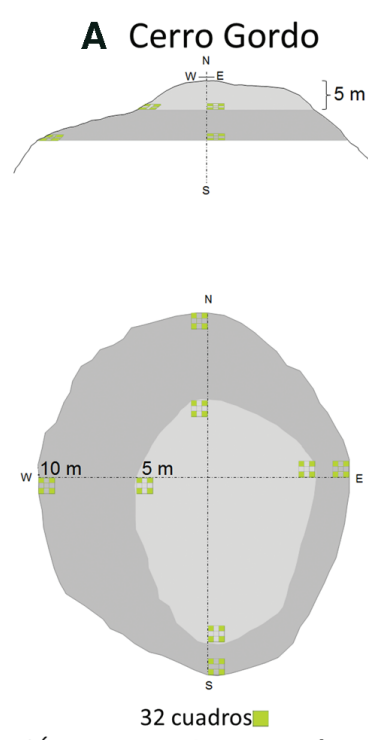

*Área aproximada $=2,809 \mathrm{~m}^{2}$
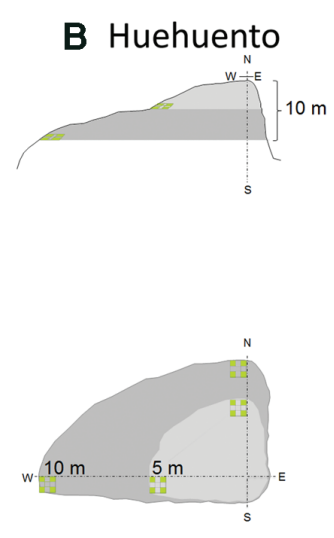

Área aproximada $=618 \mathrm{~m}^{2}$
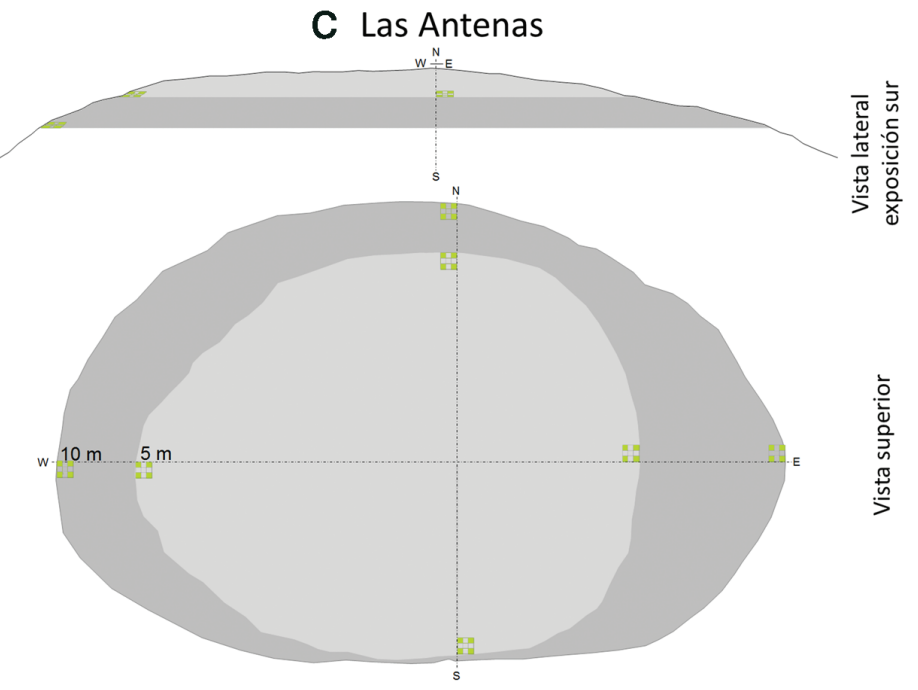

28 cuadros $\square$

Área aproximada $=8,412 \mathrm{~m}^{2}$

Unidad de muestreo $1 \mathrm{~m}^{2}=$ "cuadro"

Figura 2. Diseño esquemático de muestreo en tres cerros de la de la Sierra Madre Occidental. Área estimada hasta la curva de nivel de los -10 m desde la cima, la cual corresponde a la superficie muestreada para el inventario de especies. Los cuadros verdes corresponden a las unidades de muestreo para el análisis de correspondencia canónica.

las plantas que habitan las cimas, en el área comprendida entre el límite inferior del área cimera de los $10 \mathrm{~m}$ y la cima misma, fuera de los cuadros de muestreo. Con el fin de estimar el área total inventariada, en cada cerro se midió la distancia, a nivel de suelo, entre la cima y cada una de las parcelas de $10 \mathrm{~m}$. Se colectaron, herborizaron e identificaron ejemplares siguiendo las técnicas convencionales. La recolección se realizó de manera intensiva buscando la mayor cantidad de especies diferentes en el área de trabajo; en dicha actividad, en cada cerro participaron de cuatro a seis personas con conocimientos botánicos. Las muestras fueron depositadas en la colección del Herbario CIIDIR.

Análisis de Datos: Para evaluar la fiabilidad del esfuerzo del inventario, se realizó una curva de acumulación de especies empleando los estimadores de Chao 2, Jack 1, Jack 2 y Bootstrap, utilizando el programa EstimateS versión 8.2.0 (Colwell, 2006). Con datos de presencia/ausencia de las especies obtenidos de éste, se empleó el índice de Jaccard para medir la similitud florística entre los tres cerros. La matriz de similitud generada se convirtió a matriz de distancia (1Jaccard), y se llevó a cabo un análisis de conglomerados mediante el método UPGMA usando el programa estadístico NTSYSpc Ver.2.11x (Rohlf, 2000), esto con la finalidad de tener una representación visual de la semejanza entre los cerros por medio del dendrograma resultante.

Se construyeron dos matrices, una de 93 especies $\times 76$ cuadros con datos acerca de la cobertura de las especies, los datos fueron transformados a raíz cuadrada con la finalidad de homogeneizar la distribución y que los valores de cobertura muy altos no influyeran en la ordenación respecto a los valores mínimos de cobertura. La segunda matriz se construyó con 11 variables ambientales $\times 76$ cuadros, en la cual se incluyeron las cuatro principales variables bioclimáticas de WorldClim [temperatura mínima (Tmin), temperatura máxima (Tmax), temperatura media anual (Temp) y precipitación media anual (Prec) en la escala de 30 segundos de arco (1 km² aprox.); Hijmans et al., 2005]; así como cinco variables que se obtuvieron en campo y describen algunas características de la cima: área cimera (Area), orientación (Orie), pendiente (Pend), rocosidad (Roca) y grado de perturbación (Pert); además de la altitud de cada cima (Alt) y la continentalidad (Cont), definida como la distancia más corta de cada uno de los cerros al océano Pacífico, la cual fue obtenida a través del software ArcGIS 9.3 usando la capa de cuerpos de agua de INEGI (escala 1:1,000,000).

Se utilizó el software CANOCO versión 4.56 (ter Braak y Smilauer, 2009), con estas matrices para realizar el análisis de correspondencia canónica (ACC). Este ACC utiliza un método iterativo para relacionar variables ambientales con presencia de especies en los cuadros. Para evaluar la significancia estadística de la relación entre las variables ambientales y la presencia de especies se realizó la prueba de Permutaciones de Monte Carlo (1,000 permutaciones, $\alpha$ $=0.05)$ que se incluye en CANOCO. 


\section{Resultados}

Registros por cuadros. Dentro de los 76 cuadros muestreados se registraron un total de 93 taxa (incluidas especies no identificadas y grupos taxonómicos tratados como unidad, p. ej., musgos o helechos). En los cuadros de Cerro Gordo y Las Antenas se registraron 52 y 50 especies respectivamente, mientras que en Huehuento sólo fueron encontradas 22.

Dentro de los 32 cuadros del Cerro Gordo, las especies con mayor cobertura fueron Agrostis sp., Bromus carinatus, Galinsoga cf. subdiscoidea y Holodiscus dumosus. En los cuadros de la exposición sur se encontraron especies arbustivas que no se encontraron en ninguna otra exposición del cerro: Holodiscus dumosus y Juniperus blancoi var. huehuentensis, esta última representa una variedad arbustiva de táscate que se conocía únicamente en dos sitios de la SMO: en las cimas de los cerros Huehuento y Mohinora (Guadalupe y Calvo, Chih.). En Cerro Gordo, la masa arbórea alcanza la cima por la exposición oeste.

En el Cerro Huehuento las especies más abundantes en las exposiciones norte y oeste fueron Juniperus blancoi var. huehuentensis, Arracacia tolucensis, Aegopogon cenchroides y Holodiscus dumosus. Este cerro es el que presenta mayor superficie de roca desnuda en su cima (68.8\%). No fue posible realizar los muestreos hacia el sur y el este por presentar pendientes muy escarpadas; sin embargo, fue posible hacer observaciones y registros fotográficos en estas exposiciones. En la exposición este se aprecia la presencia de elementos arbóreos que llegan hasta aproximadamente $20 \mathrm{~m}$ debajo de la cima; el desarrollo de la vegetación en este sitio está favorecido por una cañada en dirección sur que parte del pico más alto del cerro. En la exposición sur destaca un afloramiento de roca con muy escasa cobertura vegetal.

En el cerro Las Antenas, la parcela de los $10 \mathrm{~m}$ al sur no pudo muestrearse por ser un escarpe de roca. Este cerro es el que presenta menor cobertura de roca y mayor proporción de hojarasca debido a la presencia de una mayor cubierta vegetal compuesta por varias especies de árboles y arbus- tos, entre los que destacan Arctostaphylos pungens, Holodiscus dumosus, Juniperus deppeana, Populus tremuloides y Quercus striatula, así como otras latifoliadas de talla arbórea que no se encontraron dentro de los cuadros.

Inventario florístico, riqueza específica y similitud. Se registraron un total de 175 especies distribuidas en 97 géneros y 43 familias (Apéndice 1), de las cuales las de mayor presencia son Asteraceae (42 especies), Poaceae (26) y Fabaceae (10). Aunque la curva de acumulación de especies llega a la asíntota para todos los estimadores empleados, el número de especies estimadas es siempre menor al registrado en este trabajo, siendo Jackknife 2 el que más se aproxima, con 122 especies (Figura 3).

El cerro que presentó mayor número de taxa registrados fue Las Antenas con 117 especies, lo que equivale al $67.63 \%$ de las encontradas en los tres cerros. En Cerro Gordo y Huehuento se encontraron 60 y 47 especies, respectivamente. Sin embargo, cuando se considera la riqueza de especies por superficie de muestreo, el número de especies por unidad de área es mayor en Huehuento $\left(0.07 \mathrm{spp} / \mathrm{m}^{2}\right)$ que en los demás (0.02 para el Cerro Gordo y 0.01 para Las Antenas).

En Las Antenas se encontraron 83 especies exclusivas (respecto a los otros dos cerros), mientras que solamente se encontraron 24 en el Cerro Gordo y 21 en el Huehuento. Las Antenas es también el cerro que mayor número de especies comparte con los otros dos cerros, 21 con el Cerro Gordo y 11 con el Huehuento; el Cerro Gordo y el Huehuento sólo comparten 13 especies entre sí. De las 175 especies registradas, únicamente dos se encontraron en los tres cerros.

El análisis de conglomerados confirmó la poca similitud florística entre los cerros: Cerro Gordo y Huehuento, que son los más parecidos, comparten apenas el $17 \%$ de las especies. A su vez este par de cerros comparte sólo el $12 \%$ de su flora con Las Antenas (Figura 4).

Entre los escasos elementos florísticos de afinidad alpina encontrados para la SMO están Juniperus blancoi var.

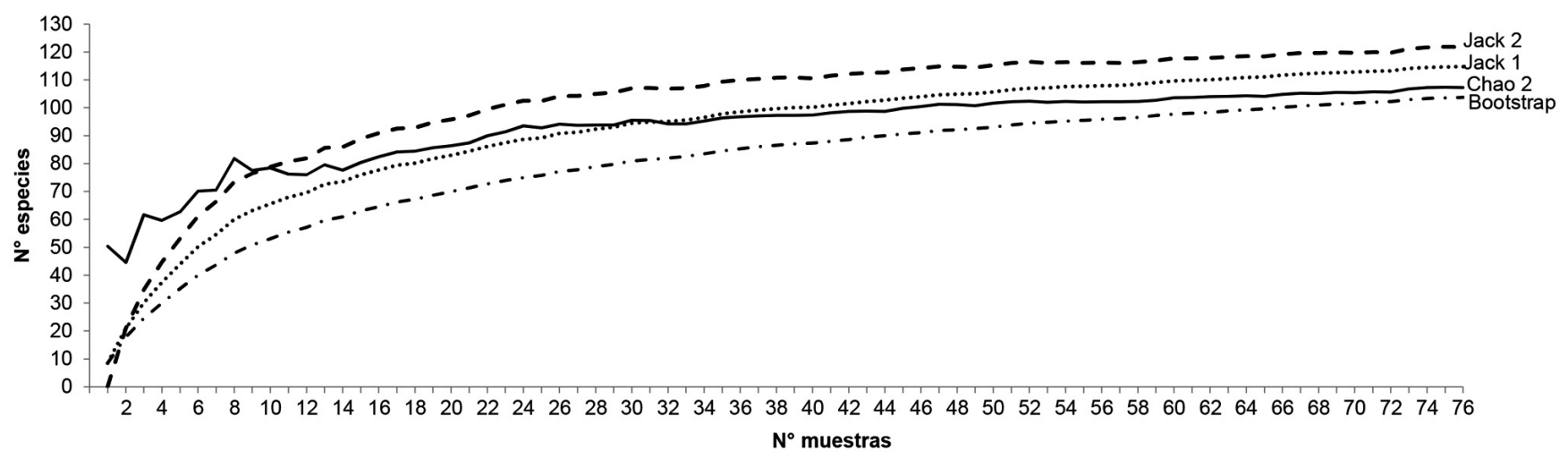

Figura 3. Curva de acumulación de especies del muestreo en tres cerros de la de la Sierra Madre Occidental. Estimación de Jack2 = 122 , Jack1 $=115$, Chao $2=107$ y Bootstrap $=104$. 

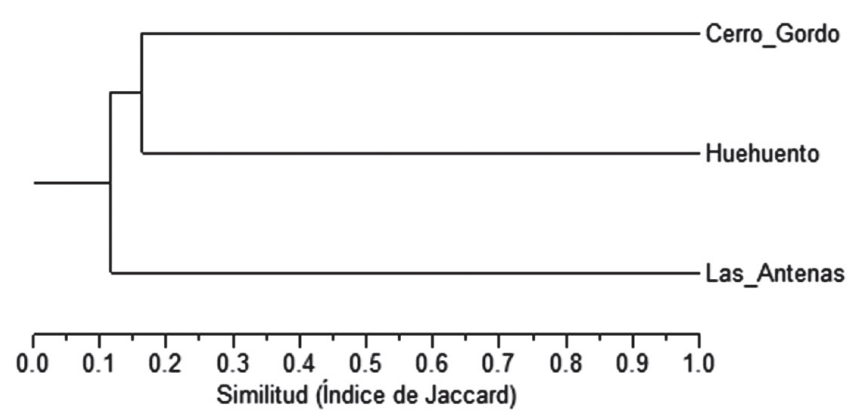

Figura 4. Dendrograma de similitud florística de tres cerros de la Sierra Madre Occidental, (UPGMA, índice de similitud de Jaccard) basado en datos de presencia-ausencia.

huehuentensis, endémico a tres cimas altas de la SMO, y las herbáceas Draba implexa, conocida sólo del Huehuento, Micranthes mexicana de Chihuahua y Durango, Bromus richardsonii, elemento boreal distribuido de Alaska a Durango, Muhlenbergia filiculmis, y varias especies de Sedum y Galinsoga que son propias de este tipo de lugares.
Relación flora-variables ambientales. La correlación especies-ambiente fue significativa $(\mathrm{F}=2.948, P=0.001)$ para los primeros cuatro ejes canónicos extraídos mediante el ACC, siendo los dos primeros los que presentan las mayores correlaciones canónicas: 0.97 para el primero y 0.93 para el segundo. Un $46.8 \%$ de la varianza de la relación entre especies y variables ambientales pudo ser explicada por los dos primeros ejes, el primero explicó un $27.8 \%$ y el segundo 19\% (Cuadro 1).

Los dos primeros ejes canónicos del ACC (Figura 5, Cuadro 1) definen un espacio en el que los sitios se ordenan, en el eje canónico I, en un gradiente de perturbación, cobertura de rocas, continentalidad, oscilación de temperatura y precipitación. De manera que los cuadros del cerro Las Antenas se encuentran en el lado negativo (más perturbado, con mayor temperatura máxima y continentalidad) y los otros dos cerros en el eje positivo (menos perturbados, con mayor cobertura de rocas, menor continentalidad, mayor precipitación y temperatura baja). El eje II está altamente relacionado en su lado positivo con la temperatura media anual

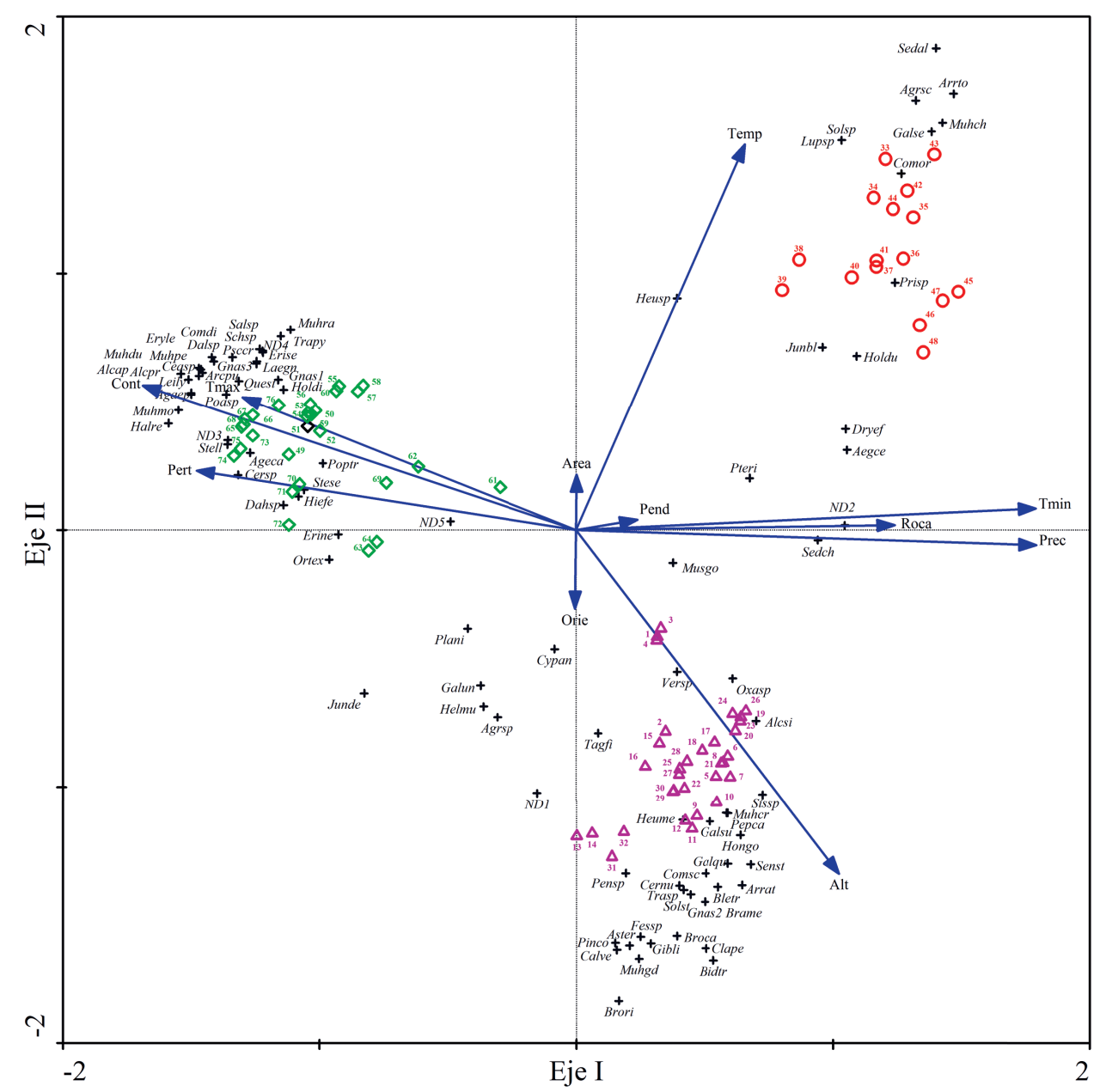

Figura 5. Diagrama de ordenación confirmatorio del CCA. Cuadros ( $\triangle$ 1-32 Cerro Gordo, $\bigcirc 33-48$ Huehuento y $\diamond 49-76$ Las Antenas), especies $(+)$ y vectores de correlación entre los ejes de ordenación y las variables ambientales $(\rightarrow)$. 
Cuadro 1. Correlaciones de los dos primeros ejes del Análisis de Correspondencia Canónica (CCA) para 76 cuadros con 93 especies de plantas y 11 variables ambientales. Se marcan en negritas los valores más altos. Inercia total: 9.74. Abreviaturas de las variables como en Material y métodos.

\begin{tabular}{lcc}
\hline & Eje 1 & Eje 2 \\
\hline Valores característicos & 0.706 & 0.482 \\
Correlación & $\mathbf{0 . 9 7 2}$ & $\mathbf{0 . 9 3 6}$ \\
especies-ambiente & & \\
Varianza acumulada (\%) & 27.8 & 46.8 \\
Correlaciones canónicas: & & \\
Area & 0.0014 & 0.1214 \\
Orie & -0.0021 & -0.1727 \\
Pend & 0.1266 & 0.0221 \\
Temp & 0.3474 & $\mathbf{0 . 8 4 4 5}$ \\
Tmax & $\mathbf{- 0 . 6 8 9 2}$ & 0.2903 \\
Tmin & $\mathbf{0 . 9 5 1 0}$ & 0.0472 \\
Prec & $\mathbf{0 . 9 5 2 2}$ & -0.0331 \\
Cont & $\mathbf{- 0 . 8 9 5 4}$ & 0.3163 \\
Alt & 0.5426 & $\mathbf{- 0 . 7 5 3 1}$ \\
Roca & $\mathbf{0 . 6 5 7 0}$ & 0.0102 \\
Pert & $\mathbf{0 . 7 6 8 2}$ & 0.1296 \\
\hline
\end{tabular}

(Temp), mientras que hacia el lado negativo la presencia de los cuadros se asocia con la elevación de los sitios. Así, los cerros Huehuento y Las Antenas son los de menor elevación y presentan una mayor temperatura media anual, en tanto Cerro Gordo es el más alto y con una temperatura media anual más baja. Las especies se asociaron con los cuadros de los cuales son exclusivas o donde presentaron una mayor cobertura.

Algunas de las especies endémicas de la SMO, como Erigeron seemanni, Juniperus blancoi var. huehuentensis y Tradescantia pygmaea, no presentan ningún patrón claro de distribución dentro de la figura. De manera consistente con la distribución de sitios, las especies generalistas e indicadoras de disturbio se encuentran del lado negativo del eje I, mientras que a la derecha se localizan varias especies que son de lugares montanos, definidos por la temperatura mínima promedio y un intervalo de precipitación más alto (Juniperus blancoi var. huehuentensis y algunas especies de los géneros Galinsoga, Lupinus, Muhlenbergia y Sedum).

\section{Discusión}

La flora de las cimas y su cobertura. Comparado con estudios similares (Kazakis et al., 2007; Eguiguren y Ojeda, 2009; Hirota et al., 2009) la riqueza específica de plantas encontradas en este estudio fue alta, teniendo en cuenta las condiciones extremas de los lugares donde se encuentran.

Las diferencias encontradas en cuanto al número de especies son el reflejo de las características particulares, tanto de cada una de las cimas como de las diferentes áreas dentro de ellas. La presencia de un fuerte afloramiento rocoso y la falta de protección que tienen las plantas en las cimas de los cerros (mayormente en el Cerro Gordo y el Huehuento), dificultan el desarrollo de especies leñosas, por lo que las formas de vida dominantes son las hierbas, plantas generalmente de talla muy pequeña, con frecuencia anuales y otras postradas o que se refugian entre las grietas de las rocas.

Aunque la presencia de especies anuales no es común en comunidades de tipo alpino; cuando ocurre, está relacionada con una marcada oscilación de la temperatura a lo largo del año (verano cálido e invierno frío), una alta radiación solar (propia de la latitud) y con un clima menos extremo (Spira y Wagner, 1988) en comparación con sitios alpinos de tipo zonal.

En cuanto a la distribución de los elementos arbóreos en las cimas, en el Cerro Gordo éstos llegan por el lado oestenoroeste, exposición que recibe la humedad proveniente de la costa y que además presenta una pendiente menos abrupta. Estos atributos permiten una mayor profundidad del suelo, favoreciendo que los individuos de Pinus rudis alcancen esa parte de la cima.

En el caso del Huehuento, los árboles más próximos a la cima se encuentran por el lado este, debido a que la topografía del sitio permite que estos elementos (Populus tremuloides y Juniperus deppeana var. robusta) se refugien de las corrientes de aire en la cañada. En Las Antenas, el que los árboles se distribuyan a lo largo de todas las exposiciones se relaciona con la forma más suave de su cima (Figura 2) y la profundidad de su suelo, aunado al hecho de que es el cerro de menor altitud.

La riqueza y similitud de las cimas y sus elementos alpinos. La baja similitud florística entre los tres cerros y las diferencias entre la heterogeneidad de especies encontrada es el reflejo de que la flora de estas cimas constituye una mezcla de diversos orígenes, en la que los distintos elementos cohabitan en un área de transición entre diferentes regiones fitogeográficas.

La mayoría de las especies registradas están distribuidas a lo largo de la SMO, y algunas se extienden desde altitudes más bajas, como el Altiplano. También existen algunas especies que llegan hasta estas cimas provenientes de Norteamérica a través de las cadenas montañosas de Arizona (Montañas Rocosas y Sky Islands), y otras más provenientes del Sur, a través de la Faja Volcánica Transmexicana.

Evidencia paleontológica muestra que algunos géneros de plantas se encontraban bien representados durante el Plioceno, y al finalizar el último periodo glaciar algunas especies permanecieron aisladas bajo condiciones ambientales muy específicas a las cuales se adaptaron (Ortega-Rosas et al., 2008 a, b). En el caso de Juniperus blancoi var. huehuentensis, que se encuentra restringido a sólo tres cerros en las partes más altas de la SMO, su diferenciación morfológica, posiblemente asociada a un hábitat frío y sin disponibilidad 
de agua corriente, puede ser considerada como una evidencia de adaptación local a este tipo de ambientes (Adams et al., 2006; Mastretta-Yanes et al., 2012).

Muchos taxa de sitios templados en México remontan su origen a poblaciones boreales que existieron durante el Pleistoceno. Los cambios climáticos ocurridos en esta Era permitieron que muchas especies migraran hacia latitudes inferiores, quedando en la actualidad distribuidas solamente dentro de los corredores montañosos, y algunas de ellas restringidas al piso alpino de las altas montañas (JaramilloCorrea et al., 2004; Moreno-Letelier y Piñero, 2009; Rodríguez-Banderas et al., 2009; Gugger et al., 2011). Estos elementos florísticos han encontrado en las asociaciones vegetales de las cimas de la SMO una manera de sobrevivir a los cambios de clima que se han dado en los últimos 100,000 años (Gugger et al., 2011); mientras que algunos elementos Neárticos son relictos de la última glaciación, otros de origen Neotropical ganan terreno hacia sitios con temperaturas menos cálidas.

El panorama general. La presencia de las especies en cada cima está estrechamente relacionada con variables bioclimáticas y con la proporción de superficie cubierta de roca. Aunque las correlaciones con el área cimera, la orientación y la pendiente de cada sitio hayan resultado bajas para el conjunto total de especies, en campo se observó que estas variables tienen una influencia local particularmente sobre ciertos taxa o individuos, que se ven favorecidos al encontrarse en lugares donde la sombra orográfica o la profundidad del suelo les ayudan a desarrollarse más fácilmente.

La riqueza, estructura y distribución de las especies de plantas estuvo relacionada con cuatro factores principales: (a) La proporción de la superficie cubierta por suelo, que en primera instancia parece tener una influencia importante, ya que el mayor número de taxa registrado fue para el cerro Las Antenas (117), que es el que presenta menor proporción de afloramiento rocoso (31\%), mientras que en el Huehuento y el Cerro Gordo se obtuvo una media de $69 \%$ y $64 \%$ de superficie cubierta por afloramiento rocoso y se registraron 47 y 60 especies, respectivamente.

Este efecto se aprecia en el diagrama de ordenación del ACC (Figura 5), en el que los cuadros de Cerro Gordo y Huehuento se encuentran en dirección a la variable "Roca" y donde se localizan las especies que en campo fueron observadas sobre afloramiento rocoso (musgo, helechos y Sedum sp.), mientras que hacia el extremo opuesto se encuentran los cuadros de Las Antenas, con suelos más profundos en los que se presenta un número mayor de especies. Sin embargo, cuando se considera el número de especies por unidad de área, la densidad de especies de Las Antenas es la menor de las tres.

(b) El relieve y la forma de cada cerro dieron pie a que el área de trabajo no fuera del mismo tamaño, ya que al delimitar las áreas hasta la curva de desnivel de los $10 \mathrm{~m}$, el grado de pendiente de cada cima determina la superficie incluida. Así, en la cima de Las Antenas, que es relativamente suave, la superficie de muestreo es más amplia y por consiguiente con mayor probabilidad de encontrar un mayor número de especies, mientras que en Huehuento, donde el relieve abrupto redujo considerablemente el área de muestreo, se registró el menor número de especies tanto en los cuadros como en el inventario.

(c) El grado de perturbación. Hobbs y Huenneke (1992) señalan que en lugares con perturbación media, la diversidad alfa puede aumentar, pues el disturbio hace que en un paisaje o comunidad se mezclen elementos de diferentes edades y etapas de la sucesión. De los tres cerros estudiados, Las Antenas es el que presenta el mayor grado de perturbación debido a la presencia de varias torres de telecomunicaciones y de una persona que vigila el lugar. Además, existen varios senderos y un camino para vehículos que permite el acceso hasta la cima del cerro.

En Las Antenas se registra la presencia de algunas especies inducidas por el fuego (Arctostaphylos pungens y Quercus striatula) y de otras que están consideradas como indicadoras de disturbio o ruderales (Lepidium virginicum, Heterosperma pinnatum, Stevia serrata y Sporobolus indicus, además de otras como Alchemilla procumbens, Agalinis peduncularis, Drymaria leptophylla, Helianthemum glomeratum y Trisetum viride, las cuales se adaptan fácilmente a diferentes hábitats (matorral, pastizal y bosque), y se distribuyen en un gradiente altitudinal más amplio (de 2,000 a 3,200 m s.n.m. o más arriba).

En Cerro Gordo y Huehuento también se encontraron especies tolerantes al disturbio como Bidens triplinervia, Muhlenbergia peruviana y Penstemon roseus, pero a diferencia del cerro Las Antenas, en estos sitios la perturbación es menor, causada por el eventual pisoteo de personas. En la cima del Cerro Gordo es frecuente la visita de grupos Huicholes que en este lugar, al que consideran sagrado, llevan a cabo ceremonias religiosas propias de su cultura. En el Huehuento es menos frecuente la presencia de personas, y la influencia sobre la vegetación está dada principalmente por herbívoros (p. ej. venados, de los cuales se observaron rastros e incluso ejemplares).

(d) La orografía regional y la elevación. El Huehuento y el Cerro Gordo presentan un mayor aislamiento de otros cerros altos, se localizan muy cerca de las barrancas y sus cimas se ven más afectadas por los vientos que llegan de manera directa hasta esos lugares, lo que provoca un efecto desecante sobre las plantas. En Huehuento, esta situación puede relacionarse con la presencia de algunos individuos secos (restos) de Juniperus deppeana var. robusta situados a pocos metros de la cima. El Cerro Gordo se ve favorecido por la corriente cálida que ofrece la quebrada del Río San Pedro-Mezquital, localizada al sur de este cerro, lo que influye para mantener una mayor estabilidad de su temperatura y permite que, pese a ser el cerro de mayor elevación 
de la SMO, alcancen la cima algunos individuos de especies arbóreas. El mayor número de especies encontrado en el cerro Las Antenas y la presencia de algunos árboles en su cima se deriva también de las condiciones menos extremas y su menor elevación, aunque también se ve afectado por un mayor efecto de continentalidad, debido a su localización en la vertiente oriental (seca) de la SMO y a su aislamiento del macizo montañoso principal.

\section{Conclusiones}

La composición florística y la diversidad de plantas varía ampliamente entre los tres cerros. La contrastante riqueza de especies entre los cerros estudiados está determinada por factores bioclimáticos, la altitud de cada cima y la proporción de rocas que cubren el suelo. Las diferencias entre los cuadros dentro de un mismo cerro están en función del lugar específico que ocupan en el cerro, la disponibilidad de suelo y el grado de disturbio son las variables más determinantes; el primero a su vez, determinado por el relieve local y por la orografía regional.

Los límites de la vegetación arbórea en la Sierra Madre Occidental son tanto de tipo zonal como azonal, determinados por condiciones climáticas locales y por el sustrato. La línea de árboles está definida en el cerro Huehuento por la ausencia de suelo y por la escasa superficie de la cima, pero en el Cerro Gordo y el de Las Antenas esta línea no existe, pues aunque algunas orientaciones sólo presenten formas de vida herbácea, unos pocos árboles llegan hasta la cima.

Se confirma la ausencia de comunidades alpinas para la SMO. Los elementos florísticos de tipo alpino son escasos. Entre ellos están Bromus richardsonii, Draba implexa, Juniperus blancoi var. huehuentensis, Micranthes mexicana, y especies de talla muy pequeña de los géneros Galinsoga, Muhlenbergia y Sedum.

Esta investigación proporciona una base de información para futuros monitoreos de la flora de cimas de la SMO, la cual es particularmente sensible al disturbio y al cambio climático. Asimismo, contribuye a aumentar el conocimiento acerca de la flora de la SMO y representa una herramienta para que el manejo de los recursos naturales se lleve a cabo de una manera más integral y con base en información confiable y actualizada. Saber con certeza cuáles son y cómo están distribuidos los elementos de una comunidad permite optimizar los planes de manejo y aprovechar de mejor manera los recursos naturales en estas áreas. Con ello, se espera que los resultados obtenidos en esta investigación sean de utilidad en la consolidación de acciones en favor de estos ecosistemas.

\section{Agradecimientos}

A Ismael Estrada, Nicolasa González, Sergio Heynes, Ezequiel Larreta, Gustavo Medrano-Valtierra, Guadalupe Nava,
Jorge Noriega, Octavio Rosales, Daniel Salinas y Abraham Torres por su apoyo en campo, así como a Félix Avitia y Ernesto Lozano por apoyo logístico. Por la identificación de algunos ejemplares a Jeffrey Bacon, Yolanda Herrera, Consuelo Medina, Emmanuel Pérez Cálix, David Ramírez, y Daniel Tejero. Y por su ayuda en gabinete y manejo de los datos a Irma Lorena López, Flor Isela Retana, Daniela Sandoval y Jorge Alberto Tena. Este estudio fue desarrollado dentro del programa de trabajo del proyecto IPN-SIP 20110681, y forma parte del trabajo de tesis de maestría (CIIDIR IPN Durango) de LRG. Agradecemos a Eduardo Estrada y un revisor anónimo por sus recomendaciones, las cuales ayudaron a mejorar este trabajo.

\section{Literatura citada}

Adams R.P., González-Elizondo M.S., González-Elizondo M. y Slinkman E. 2006. DNA fingerprinting and terpenoid analysis of Juniperus blancoi var. huehuentensis (Cupressaceae), a new subalpine variety from Durango, Mexico. Biochemical Systematics and Ecology 34:205-211.

Colwell R.K. 2006. EstimateS v. 8.2.0. Statistical estimation of species richness and shared species from samples. <http://www. purl.oclc.org/estimates $>$ (consultado 8 agosto 2012).

Correll D.S. 1960. A mule-train trip to Sierra Mohinora, Chihuahua. American Fern Journal 50:66-78.

Eguiguren P. y Ojeda T. 2009. Línea base para el monitoreo a largo plazo del impacto del cambio climático, sobre la diversidad florística en una zona piloto del ecosistema páramo del Parque Nacional Podocarpus. Tesis de licenciatura, Carrera de Ingeniería Forestal, Universidad Nacional de Loja, Quito. 101 pp.

Giménez J., Ramírez M. I. y Pinto M. 2003. Las comunidades vegetales de la Sierra de Angangueo (estados de Michoacán y México, México): clasificación, composición y distribución. Lazaroa 24:87-111.

González-Elizondo M.S., González-Elizondo M. y Márquez-Linares M. A. 2007. Vegetación y Ecorregiones de Durango. Plaza y Valdés Editores-Instituto Politécnico Nacional. México, D.F.

González-Elizondo M.S., González-Elizondo M., Tena-Flores J.A., Ruacho-González L. y López-Enríquez I.L. 2012. Vegetación de la Sierra Madre Occidental, México: Una síntesis. Acta Botanica Mexicana 100:351-403.

Grabherr G., Gottfried M. y Pauli H. 2010. Climate change impacts in alpine environments. Geography Compass 4:1133-1153.

Hijmans R.J., Cameron S.E., Parra J.L., Jones P.G. y Jarvis A. 2005. Very high resolution interpolated climate surfaces for global land areas. International Journal of Climatology 25:1965-1978.

Hirota M., Zhang P., Gu S., Du M., Shimono A., Shen H., Li Y. y Tang Y. 2009. Altitudinal variation of ecosystem $\mathrm{CO}_{2}$ fluxes in an alpine grassland from 3600 to $4200 \mathrm{~m}$. Journal of Plant Ecology 2:197-205.

Hobbs R.J. y Huenneke L.F. 1992. Disturbance, diversity, and invasion: implications for conservation. Conservation Biology 6:324-337.

Kazakis G., Ghosn D., Vogiatzakis N. y Papanastasis P. 2007. Vascular plant diversity and climate change in the alpine zone of the Lefka Ori, Crete. Plant Conservation and Biodiversity 16:1603-1615. 
Mastretta-Yanes A., Wegier A., Vázquez-Lobo A. y Piñero D. 2012. Distinctiveness, rarity and conservation in a subtropical highland conifer. Conservation Genetics 13:211-222.

McDonald J.A. 1993. Phytogeography and history of the alpinesubalpine flora of the northeastern Mexico. En: Ramamoorthy T.P., Bye R., Lot A. y Fa J. Eds. Biological Diversity of Mexico: Origins and Distribution, pp. 681-703, Oxford University Press, Nueva York.

McDonald J.A., Martínez J. y Nesom G.L. 2011. Alpine flora of Cerro Mohinora, Chihuahua, Mexico. Journal of the Botanical Research Institute of Texas 5:701-705.

Morrone J.J. 2005. Hacia una síntesis biogeográfica de México. Revista Mexicana de Biodiversidad 76:207-252.

Nagy L. y G. Grabherr. 2009. The Biology of Alpine Habitats. Oxford University Press, Nueva York.

Ortega-Rosas C.I., Peñalba M.C., López-Sáez J.A. y Van Devender T.R. 2008a. Retrospectiva del bosque de pino y encino de la Sierra Madre Occidental, Sonora, noroeste de México, hace 1000 años. Acta Botanica Mexicana 83:69-92.

Ortega-Rosas C.I., Peñalba M.C. y Guiot J. 2008b. Holocene altitudinal shifts in vegetation belts and environmental changes in the Sierra Madre Occidental, Northwestern Mexico, based on modern and fossil pollen data. Review of Palaeobotany and Palynology 151:1-20.

Pauli H., Gottfried M., Hohenwallner D., Reiter K., Grabherr G. y Villar L. 2003. Manual para el trabajo de campo del Proyecto GLORIA. Iniciativa para la investigación y el seguimiento global de los ambientes alpinos, como contribución al sistema terrestre de observación global (GTOS). Universidad de Viena e Instituto Pirenaico de Ecología, Huesca.

Rohlf F.J. 2000. NTSYSpc: numerical taxonomy and multivariate analysis system. Version 2.11x. Applied Biostatistics Inc, Nueva York.

Ruacho-González L. 2011. El elemento alpino en la vegetación de cimas de la Sierra Madre Occidental. Tesis de maestría. Centro Interdisciplinario de Investigación para el Desarrollo Integral Regional, Instituto Politécnico Nacional. Durango. 83 pp.

Rzedowski J. 1978. La Vegetación de México. Limusa, México, D.F.

Spira T.P. y Wagner L. K. 1988. Weedy annuals in the alpine zone of the White Mountains, California. En: Hall C.A. y DoyleJones V. Eds. Plant Biology of Eastern California, pp. 92-98, White Mountain Research Station, University of California, Los Angeles.

ter Braak C.J.F. y Smilauer P. 2009. CANOCO for Windows version 4.56. Biometrics-Plant Research International, Wageningen.

Villar L. y Benito J.L. 2003. La flora alpina de Europa y el cambio climático: El caso del Pirineo Central. En: Asociación Española de Ecología Terrestre. Ed. España ante los Compromisos del Protocolo de Kyoto: Sistemas Naturales y Cambio Climático. VII Congreso Nacional de la Asociación Española de Ecología Terrestre, pp. 92-105, Barcelona.

Wieser G., Matyssek R., Luzian R., Zwerger P., Pindur P., Oberhuber W. y Gruber A. 2009. Effect of atmospheric and climate change at the timberline of the Central European Alps. Annals of Forest Science 66:402.

Recibido: 17 de mayo de 2012

Aceptado: 9 de octubre de 2012 
Apéndice 1. Listado florístico.

\begin{tabular}{|c|c|c|c|c|c|c|c|}
\hline Especie & $\begin{array}{l}\text { Cerro } \\
\text { Gordo }\end{array}$ & Huehuento & Antenas & Especie & $\begin{array}{l}\text { Cerro } \\
\text { Gordo }\end{array}$ & Huehuento & Antenas \\
\hline TRACHEOPHYTA & & & & Iridaceae & & & \\
\hline MONILOPHYTA & & & & Orthrosanthus exsertus (R.C. & - & - & $x$ \\
\hline Athyriaceae & & & & Foster) Ravenna & & & \\
\hline Woodsia mollis (Kaulf.) J.Sm. & - & - & $x$ & Sisyrinchium arizonicum Rothr. & - & $x$ & - \\
\hline Woodsia phillipsii Windham & - & $x$ & - & Sisyrinchium cernuum (E.P. & - & $x$ & $x$ \\
\hline Pteridaceae & & & & Bicknell) Kearney & & & \\
\hline Cheilanthes pyramidalis Fée & $x$ & - & $x$ & Sisyrinchium sp. & $x$ & - & - \\
\hline Pellaea ternifolia (Cav.) Link & - & - & $x$ & Juncaceae & & & \\
\hline subsp. ternifolia & & & & $\begin{array}{l}\text { Juncus tenuis Willd. } \\
\text { Melanthiaceae }\end{array}$ & - & - & $x$ \\
\hline SPERMATOPHYTA & & & & Schoenocaulon sp. & - & - & $x$ \\
\hline GIMNOSPERMAE & & & & Orchidaceae & & & \\
\hline Cupressaceae & & & & Dichromanthus michuacanus & - & - & $x$ \\
\hline $\begin{array}{l}\text { Juniperus blancoi Martínez } \\
\text { var. huehuentensis R.P.Adams, }\end{array}$ & $x$ & $x$ & - & $\begin{array}{l}\text { (La Llave \& Lex.) Salazar \& } \\
\text { Soto Arenas }\end{array}$ & & & \\
\hline S.González \& M.González & & & & Habenaria guadalajarana S.Watson & - & - & $x$ \\
\hline Juniperus deppeana Steud. s.I. & $x$ & - & $x$ & Malaxis soulei L.O.Williams & - & - & $x$ \\
\hline Pinaceae & & & & Poaceae & & & \\
\hline Pinus rudis Endl. & $x$ & - & $x$ & $\begin{array}{l}\text { Aegopogon cenchroides Humb. } \\
\text { \& Bonpl. ex Willd. }\end{array}$ & $x$ & $x$ & - \\
\hline ANGIOSPERMAE & & & & Aegopogon tenellus (DC.) Trin. & - & - & $x$ \\
\hline LILIOPSIDA & & & & Agrostis liebmannii (E.Fourn.) & - & - & $x$ \\
\hline Agavaceae & & & & Hitchc. & & & \\
\hline Prochnyanthes mexicana Rose & - & - & $x$ & Agrostis scabra Willd. & - & $x$ & - \\
\hline Amaryllidaceae & & & & Agrostis sp. & $x$ & - & - \\
\hline $\begin{array}{l}\text { Allium sp. } \\
\text { Calochortaceae }\end{array}$ & - & - & $x$ & $\begin{array}{l}\text { Blepharoneuron tricholepis } \\
\text { (Torr.) Nash }\end{array}$ & $x$ & $x$ & $x$ \\
\hline $\begin{array}{l}\text { Calochortus venustulus Greene } \\
\text { var. venustulus }\end{array}$ & $x$ & - & - & $\begin{array}{l}\text { Brachypodium mexicanum } \\
\text { (Roem. \& Schult.) Link }\end{array}$ & $x$ & - & $x$ \\
\hline Commelinaceae & & & & Bromus carinatus Hook. \& Arn. & $x$ & $x$ & - \\
\hline Commelina dianthifolia Delile & - & - & $x$ & var. californicus Shear & & & \\
\hline Commelina orchioides Booth & - & $x$ & - & Bromus richardsonii Link & $x$ & - & - \\
\hline ex Lindl. & & & & Festuca pringlei St.-Yves & - & - & $x$ \\
\hline Commelina scabra Benth. & $x$ & - & - & Festuca rubra L. & - & - & $x$ \\
\hline Gibasis linearis (Benth.) & $x$ & - & - & Festuca sp. & $x$ & - & $x$ \\
\hline Rohweder subsp. linearis & & & & Muhlenbergia crispiseta Hitchc. & $x$ & - & - \\
\hline Tradescantia pygmaea D.R.Hunt & $x$ & - & $x$ & Muhlenbergia dubia E.Fourn. & - & - & $x$ \\
\hline Cyperaceae & & & & Muhlenbergia filiculmis Vasey & - & $x$ & - \\
\hline Carex turbinata Liebm. s.I. & $x$ & - & - & Muhlenbergia montana Hitchc. & - & $x$ & $x$ \\
\hline Cyperus cf. andinus Palla ex Kük. & $x$ & - & $x$ & $\begin{array}{l}\text { Muhlenbergia peruviana } \\
\text { (P.Beauv.) Steud. }\end{array}$ & - & $x$ & $x$ \\
\hline
\end{tabular}


Diversidad florística en cimas de la Sierra Madre Occidental

Apéndice 1. Continuación.

\begin{tabular}{|c|c|c|c|c|c|c|c|}
\hline Especie & $\begin{array}{l}\text { Cerro } \\
\text { Gordo }\end{array}$ & Huehuento & Antenas & Especie & $\begin{array}{l}\text { Cerro } \\
\text { Gordo }\end{array}$ & Huehuento & Antenas \\
\hline Muhlenbergia ramulosa & - & $x$ & $x$ & Pseudognaphalium sp. 3 & - & $x$ & $x$ \\
\hline (Kunth) Swallen & & & & Pseudognaphalium sp. 4 & - & - & $x$ \\
\hline Muhlenbergia vaginata Swallen & - & - & $x$ & Pseudognaphalium sp. 5 & - & - & $x$ \\
\hline $\begin{array}{l}\text { Muhlenbergia virescens } \\
\text { (Kunth) Trin. }\end{array}$ & - & - & $x$ & $\begin{array}{l}\text { Gutierrezia alamanii A. Gray } \\
\text { var. megalocephala (Fernald) }\end{array}$ & - & $x$ & - \\
\hline Peyritschia pringlei (Scribn.) & - & - & $x$ & M.A.Lane & & & \\
\hline S.D.Koch & & & & Heliomeris multiflora Nutt. s.I. & $x$ & - & $x$ \\
\hline Poa annua L. & - & - & $x$ & Heliomeris multiflora Nutt. & - & - & $x$ \\
\hline Sporobolus indicus (L.) R.Br. & - & - & $x$ & var. nevadensis (A.Nelson) & & & \\
\hline Trisetum palmeri Hitchc. & - & - & $x$ & W.F.Yates & & & \\
\hline Trisetum viride Kunth & - & - & $x$ & Heterosperma pinnatum Cav. & - & - & $x$ \\
\hline sp. & - & - & $x$ & $\begin{array}{l}\text { Hieracium aff. dysonymum } \\
\text { S.F.Blake }\end{array}$ & $x$ & - & - \\
\hline MAGNOLIOPSIDA & & & & Hieracium fendleri Sch.Bip. & $x$ & - & $x$ \\
\hline Apiaceae & & & & Jaegeria hirta (Lag.) Less. & - & - & $x$ \\
\hline $\begin{array}{l}\text { Arracacia aff. atropurpurea } \\
\text { Benth. \& Hook.f. ex Hemsl. }\end{array}$ & $x$ & - & - & $\begin{array}{l}\text { Laennecia confusa (Cronquist) } \\
\text { G.L.Nesom }\end{array}$ & - & - & $x$ \\
\hline Arracacia tolucensis Hemsl. & - & $x$ & - & Laennecia gnaphalioides & - & - & $x$ \\
\hline Eryngium globosum Hemsl. & - & - & $x$ & (Kunth) Cass. & & & \\
\hline $\begin{array}{l}\text { Eryngium lemmonii J.M.Coult. } \\
\quad \& \text { Rose }\end{array}$ & - & - & $x$ & $\begin{array}{l}\text { Laennecia schiedeana (Less.) } \\
\text { G.L.Nesom }\end{array}$ & - & - & $x$ \\
\hline Asteraceae & & & & Leibnitzia lyrata (Sch.Bip) & - & - & $x$ \\
\hline Ageratina calaminthifolia (Kunth) & - & - & $x$ & G.L.Nesom & & & \\
\hline R.M.King \& H.Rob. & & & & Psacalium cronquistiorum & - & - & $x$ \\
\hline Bidens heterosperma A.Gray & - & - & $x$ & B.L.Turner & & & \\
\hline Bidens triplinervia Kunth & $x$ & - & - & Roldana hartwegii (Benth.) H.Rob. & - & $x$ & $x$ \\
\hline $\begin{array}{l}\text { Brickellia oreithales (B.L.Rob.) } \\
\text { Shinners }\end{array}$ & - & - & $x$ & $\begin{array}{l}\text { \& Brettell var. durangensis } \\
\text { (H.Rob. \& Brettell) Funstron }\end{array}$ & & & \\
\hline Carphochaete durangensis & - & - & $x$ & Senecio sp. & $x$ & - & - \\
\hline Grashoff ex B.L.Turner & & & & Senecio stoechadiformis DC. & $x$ & - & - \\
\hline Cirsium sp. & - & - & $x$ & Senecio toluccanus DC. & - & $x$ & $x$ \\
\hline Dahlia sp. & - & - & $x$ & Stevia viscida Kunth & - & - & $x$ \\
\hline Erigeron neomexicanus A.Gray & $x$ & - & $x$ & Stevia serrata Cav. & $x$ & - & $x$ \\
\hline Erigeron seemannii Greene & - & - & $x$ & Tagetes filifolia Lag. & $x$ & - & $x$ \\
\hline Galinsoga cf. quadriradiata & $x$ & - & - & Tagetes micrantha Cav. & - & $x$ & - \\
\hline Ruiz \& Pav. & & & & Verbesina sp. & $x$ & - & - \\
\hline Galinsoga cf. semicalva (A.Gray) & - & $x$ & $x$ & sp. 1 & - & - & $x$ \\
\hline H.St.John \& D.White & & & & sp. 2 & - & - & $x$ \\
\hline Galinsoga cf. subdiscoidea & $x$ & $x$ & - & sp. 3 & - & - & $x$ \\
\hline Cronquist & & & & sp. 4 & - & - & $x$ \\
\hline Pseudognaphalium sp. 1 & - & $x$ & $x$ & & & & \\
\hline Pseudognaphalium sp. 2 & $x$ & - & - & & & & \\
\hline
\end{tabular}


Apéndice 1. Continuación.

\begin{tabular}{|c|c|c|c|c|c|c|c|}
\hline Especie & $\begin{array}{l}\text { Cerro } \\
\text { Gordo }\end{array}$ & Huehuento & Antenas & Especie & $\begin{array}{l}\text { Cerro } \\
\text { Gordo }\end{array}$ & Huehuento & Antenas \\
\hline Brassicaceae & & & & Fagaceae & & & \\
\hline Draba implexa Rollins & - & $x$ & - & Quercus depressipes Trel. & - & - & $x$ \\
\hline Lepidium virginicum L. & - & - & $x$ & Quercus sideroxyla Humb. & - & - & $x$ \\
\hline Pennellia longifolia (Benth.) & - & $\mathrm{x}$ & - & \& Bonpl. & & & \\
\hline Cactaceae & & & & Gentianaceae & & & \\
\hline Mammillaria moelleriana Boed. & - & - & $x$ & Gentianella amarella (L.) Börner & - & $x$ & - \\
\hline $\begin{array}{l}\text { Mammillaria senilis Lodd. } \\
\text { ex Salm-Dyck }\end{array}$ & - & - & $x$ & $\begin{array}{l}\text { Gentianella amarella (L.) Börner } \\
\text { subsp. mexicana (Griseb.) }\end{array}$ & - & - & $x$ \\
\hline Drymaria effusa A.Gray & $x$ & $x$ & - & Erodium cicutarium (L.) L'Hér. & - & - & $x$ \\
\hline $\begin{array}{l}\text { Drymaria leptophylla (Cham. \& } \\
\text { Schltdl.) Fenzl ex Rohrb }\end{array}$ & - & - & $x$ & $\begin{array}{l}\text { Geranium aff. atropurpureum } \\
\text { A.Heller }\end{array}$ & - & - & $x$ \\
\hline Drymaria sp. & - & - & $x$ & Grossulariaceae & & & \\
\hline Cistaceae & & & & Ribes sp. & - & $x$ & - \\
\hline Helianthemum glomeratum Lag. & - & - & $x$ & Lamiaceae & & & \\
\hline Crassulaceae & & & & Agastache eplingiana R.W. & $\mathrm{x}$ & - & $x$ \\
\hline Sedum sp. 1 & - & $x$ & - & Sanders & & & \\
\hline Sedum sp. 2 & $x$ & $x$ & $x$ & Monarda citriodora Cerv. ex & - & - & $x$ \\
\hline Fabaceae & & & & Pennell & & & \\
\hline Astragalus ervoides Hook. & - & $x$ & - & Castilleja aspera Eastw. & - & - & $x$ \\
\hline \& Arn. s.l. & & & & Castilleja saltensis Eastw. & - & $x$ & - \\
\hline $\begin{array}{l}\text { Astragalus ervoides Hook. \& Arn. } \\
\text { var. maysillesii Barneby }\end{array}$ & $x$ & - & - & $\begin{array}{l}\text { sp. } \\
\text { Oxalidaceae }\end{array}$ & - & $x$ & - \\
\hline Astragalus pennellianus Barneby & $x$ & $x$ & - & Oxalis aff. alpina Rose & - & - & $x$ \\
\hline Astragalus sp. & $\mathrm{x}$ & - & - & Oxalis sp. 1 & $\mathrm{x}$ & - & - \\
\hline Dalea cyanea Greene & - & - & $x$ & Oxalis sp. 2 & $x$ & $x$ & - \\
\hline Dalea thouinii Schrank & $x$ & - & - & Piperaceae & & & \\
\hline Dalea sp. & - & - & $x$ & Peperomia campylotropa & $x$ & - & - \\
\hline Lupinus cf. huachucanus & - & - & $x$ & A.W.Hill. & & & \\
\hline M.E.Jones & & & & Plantaginaceae & & & \\
\hline Lupinus sp. & - & $x$ & $x$ & Plantago alismatifolia Pilg. & - & $x$ & - \\
\hline Trifolium sp. 1 & - & - & $x$ & Plantago nivea Kunth & $x$ & - & $x$ \\
\hline
\end{tabular}


Apéndice 1. Continuación.

\begin{tabular}{|c|c|c|c|c|c|c|c|}
\hline Especie & $\begin{array}{l}\text { Cerro } \\
\text { Gordo }\end{array}$ & Huehuento & Antenas & Especie & $\begin{array}{l}\text { Cerro } \\
\text { Gordo }\end{array}$ & Huehuento & Antenas \\
\hline Polygalaceae & & & & Saxifragaceae & & & \\
\hline Polygala sp. & - & - & $x$ & Heuchera cf. mexicana W.Schaffn. & $x$ & - & - \\
\hline Portulacaceae & & & & Heuchera sp. & - & $x$ & $x$ \\
\hline Claytonia perfoliata Donn ex & $x$ & $x$ & - & Micranthes mexicana (Engl. \& & - & $x$ & - \\
\hline Willd. subsp. mexicana (Rydb.) & & & & Irmsch) Brouillet \& Gornall & & & \\
\hline John M.Mill \& K.L.Chambers & & & & Solanaceae & & & \\
\hline Primulaceae & & & & Solanum demissum Lindl. & - & $x$ & - \\
\hline Primula rusbyi Greene & $x$ & $x$ & - & Solanum stoloniferum Schltdl. & $x$ & - & - \\
\hline Rhamnaceae & & & & Veronicaceae & & & \\
\hline Ceanothus sp. & - & - & $x$ & Penstemon campanulatus Willd. & $x$ & - & - \\
\hline Rosaceae & & & & subsp. campanulatus & & & \\
\hline Alchemilla aphanoides Mutis. & - & - & $x$ & Penstemon roseus G.Don & $x$ & - & $x$ \\
\hline Alchemilla procumbens Rose & - & - & $x$ & Penstemon sp. & $x$ & - & $x$ \\
\hline var. procumbens & & & & Veronica peregrina L. subsp. & - & - & $x$ \\
\hline Alchemilla sibbaldiifolia Kunth & $x$ & $x$ & - & xalapensis (Kunth) Pennell & & & \\
\hline Holodiscus discolor (Pursh) & - & - & $x$ & No Identificadas & & & \\
\hline Maxim. & & & & sp. 1 & $x$ & - & $x$ \\
\hline Holodiscus dumosus (S.Watson.) & $x$ & $x$ & - & sp. 2 & $x$ & $x$ & - \\
\hline A.Heller & & & & sp. 3 & - & - & $x$ \\
\hline Rubiaceae & & & & sp. 4 & - & & $x$ \\
\hline Galium uncinulatum DC. & $x$ & - & $x$ & sp. 5 & $x$ & - & $x$ \\
\hline Salicaceae & & & & sp. 6 & - & - & $x$ \\
\hline \multirow[t]{2}{*}{ Populus tremuloides Michx. } & $x$ & - & $x$ & sp. 7 & - & - & $x$ \\
\hline & & & & sp. 8 & - & $x$ & - \\
\hline
\end{tabular}

